\title{
ON THE DYNAMICS OF TROJAN ASTEROIDS
}

\author{
B. ÉRDI \\ Department of Astronomy \\ Eötvös University, Budapest, Hungary
}

\begin{abstract}
The author's theory of Trojan asteroids (Érdi, 1988) is developed further. The motion of the Trojans is considered in the framework of the three-dimensional elliptic restricted three-body problem of the Sun-Jupiter-asteroid system including also the secular changes of Jupiter's orbital eccentricity and the apsidal motion of Jupiter's elliptic orbit. An asymptotic solution is derived, by applying the multiple-timescale method, for the cylindrical coordinates of the asteriods and for the perturbations of the orbital elements. This solution is used for the analysis of the long-time dynamical behaviour of the perihelion and the eccentricity of the Trojans.
\end{abstract}

\section{Introduction}

The Trojan asteroids have very complex dynamical behaviour, and the need for understanding their motions has stimulated many research efforts. In a series of papers Garfinkel (1985, and references therein) studied the planar motion of the Trojans in the model of the circular restricted three-body problem using the Hori-Lie perturbation method. His theory was extended to the spatial case by Zagretdinov (1986). Significant acheivements were obtained by Bien and Schubart (1987) who derived proper elements for 41 Trojans: proper eccentricity, proper inclination, and a parameter which characterizes the amplitude of libration around the Lagrangian points $L_{4}$ or $L_{5}$. Milani (1993) extended this work and computed proper elements for 174 Trojans by integrating the orbits for 1 million years and considering the perturbations of the four outer major planets. The proper elements, being almost constant for a very long time, allowed Milani to search for families of Trojan asteroids and also to establish the dynamical structure of the Trojan cloud. Despite all these efforts many problems of the Trojans 
have remained open. Such unsolved problems are (Milani, 1994): the origin of chaos in the orbits of Trojans, the question of the stability boundaries of the Trojan cloud, the origin of the orbital distribution (high inclinations) of the Trojans.

Unfortunately, existing analytical theories cannot give answers for these questions, they only contribute to the understanding of some basic features of the dynamics of the Trojans. I developed an approximate analytical theory (Érdi, 1981, 1984, 1988) which has been useful in analyzing some long periodic perturbations. This theory is based on the model of the three-dimensional elliptic restricted three-body problem of the Sun-Jupiterasteroid system with Jupiter moving in a fixed elliptic orbit. Since in the motions of the Trojans there are important perturbations coming from the other major planets too, it would be necessary to develop an analytical theory which accounts for these perturbations. In this paper a step is made in this direction, a part of the effects of the other planets is taken into consideration. Namely, it is assumed that the orbit of Jupiter is secularly changing due to the perturbations of the other planets. Thus the motion of the Trojans is considered in the framework of the three-dimensional elliptic restricted three-body problem of the Sun-Jupiter-asteroid system with Jupiter moving in an elliptic orbit whose eccentricity and perihelion are secularly changing.

\section{A Theory of Trojan Asteroids}

The following equations of motion can be derived for the Trojans:

$$
\begin{aligned}
& \frac{d^{2} r}{d v^{2}}-r\left(\frac{d \alpha}{d v}\right)^{2}-2 r \frac{d \alpha}{d v}-2 \beta r\left(\frac{d \alpha}{d v}+1\right)= \\
& \frac{1}{1+e_{J} \cos v}\left[r-\frac{1-\mu}{R_{1}^{3}} r+\mu\left(\frac{\cos \alpha-r}{R_{2}^{3}}-\cos \alpha\right)\right] \\
& \frac{d}{d v}\left(r^{2} \frac{d \alpha}{d v}+r^{2}\right)+2 \beta r \frac{d r}{d v}=\frac{\mu r \sin \alpha}{1+e_{J} \cos v}\left(1-\frac{1}{R_{2}^{3}}\right), \\
& \frac{d^{2} z}{d v^{2}}+z=\frac{z}{1+e_{J} \cos v}\left(1-\frac{1-\mu}{R_{1}^{3}}-\frac{\mu}{R_{2}^{3}}\right), \\
& R_{1}=\left(r^{2}+z^{2}\right)^{1 / 2}, \quad R_{2}=\left(1+r^{2}-2 r \cos \alpha+z^{2}\right)^{1 / 2}
\end{aligned}
$$

Here $r$ and $\alpha$ are the polar coordinates of the asteroid in the orbital plane of Jupiter ( $r$ is the distance from the Sun, $\alpha$ is the angle between the asteroid and Jupiter), $z$ is the perpendicular distance of the asteroid from the orbital plane of Jupiter, $v$ is the true anomaly of Jupiter which serves as independent variable, $e_{J}$ is the eccentricity of Jupiter's orbit, $\mu=m_{J} /\left(m_{S}+m_{J}\right)$, $m_{S}, m_{J}$ being the mass of the Sun and Jupiter. The coordinates $r$ and $z$ 
are dimensionless, the instantaneous Sun-Jupiter distance serving as unit distance. The parameter $\beta$ is connected with the precession of the orbit of Jupiter: $\beta=\dot{\varpi}_{J} / n_{J}, \dot{\varpi}_{J}$ is the secular rate of the motion of the perihelion of Jupiter's orbit (the dot means differentiation with respect to the time) and $n_{J}$ is the mean motion of Jupiter. At the derivation of Equations (1) it has been assumed that the orbit of Jupiter is a precessing ellipse for which the relations

$$
R_{J}=\frac{a_{J}\left(1-e_{J}^{2}\right)}{1+e_{J} \cos v}, \quad R_{J}^{2} \dot{v}=\sqrt{k^{2}\left(m_{S}+m_{J}\right) a_{J}\left(1-e_{J}^{2}\right)}
$$

are valid with slowly changing $e_{J}$ eccentricity $\left(a_{J}\right.$ is the semi-major axis of the orbit of Jupiter, $k$ is the Gaussian gravitational constant). Equations (1) are only approximate, terms depending on $\dot{\varpi}_{J}^{2}, \ddot{\varpi}_{J}, \dot{e}_{J} / n_{J}$ have been neglected. Equations (1) are accurate up to the third order of the small parameter $\varepsilon=\sqrt{\mu}=0.030885$. The parameter $\beta$ is of order $\varepsilon^{3}$, since the mean value of $\dot{\varpi}_{J}$ is 4.2 "/year and $n_{J}=299.1$ "/day.

The solution of Equations (1) was studied earlier (Érdi, 1981, 1984, 1988 ) for the case $\beta=0, e_{J}=$ const $=0.048$. Thus the question now is how do the new assumptions $\left(\beta \neq 0, e_{J}\right.$ changing $)$ modify the previous solution.

Since the basic periods of the Trojans are well separated, Equations (1) are suitable for the application of the method of the multiple-variable expansions. A two-variable solution for the planar motion of the Trojans in the circular restricted three-body problem was first derived by Kevorkian (1970). According to the paper (Érdi, 1984), the solution of Equations (1) can be assumed in the form of a four-variable asymptotic expansion

$$
\begin{aligned}
& r=1+\sum_{n=1}^{N} \varepsilon^{n} r_{n}(v, u, \tau, w)+O\left(\varepsilon^{N+1}\right), \\
& \alpha=\alpha_{0}(u, \tau, w)+\sum_{n=1}^{N} \varepsilon^{n} \alpha_{n}(v, u, \tau, w)+O\left(\varepsilon^{N+1}\right), \\
& z=\varepsilon^{1 / 2}\left[\sum_{n=0}^{N} \varepsilon^{n} z_{n}(v, u, \tau, w)+O\left(\varepsilon^{N+1}\right)\right],
\end{aligned}
$$

where $u=\varepsilon\left(v-v_{0}\right), \tau=\varepsilon^{2}\left(v-v_{0}\right), w=\varepsilon^{3}\left(v-v_{0}\right)$ and $v_{0}$ is the epoch. The four variables correspond to the time-scales of the motions of the asteroids, that is to the revolution around the Sun, to the libration around the Lagrangian points, to the motion of the perihelion, and to the motion of the orbital plane. In addition to (2), it will be assumed that in Equations (1)

$$
e_{J}=\varepsilon e_{1}(w), \quad \beta=\varepsilon^{3} \beta_{1}(w),
$$


where $e_{1}$ and $\beta_{1}$ are not very large in absolute value compared to unity, and they are changing on the slowest time-scale.

The substitution of (2) into Equations (1) result in a system of partial differential equations for the unknown functions $r_{n}, \alpha_{n}, z_{n}$ with independent variables $v, u, \tau$, and $w$. In the solution of this system arbitrary functions of the independent variables appear. These are specified according to the principle that the solution for $r, \alpha, z$ be bounded. The solution to $O\left(\varepsilon^{2}\right)$ was derived in Érdi (1981), and the solution in the third order was studied in Érdi (1984).

I have recalculated this earlier solution with the new terms in Equations (1) and with the conditions (3). The terms depending on $\beta$ modify the solution starting from the third order. The changing of $e_{J}$ has effects already in the first order. From the solution for the coordinates $r, \alpha, z$, the perturbations of the orbital elements can be derived by means of the relations of the two-body problem. In the following, perturbations of the eccentricity $e$ and of the longitude of the perihelion $\varpi$ of the Trojan asteroids are discussed.

\section{Long Term Perturbations of $e$ and $\varpi$}

Without the terms depending on the two fastest variables $v$ and $u$, the main perturbations of $O(\varepsilon)$ in $e$ and $\varpi$ are:

$$
\begin{aligned}
& e \cos \left(\varpi-\varpi_{J}\right)=(b-c \cos \chi)\left(1+\frac{1}{4} \kappa+\frac{5}{8} \kappa \cos \gamma\right)+(a-c \sin \chi) \frac{5}{8} \kappa \sin \gamma, \\
& e \sin \left(\varpi-\varpi_{J}\right)=(a-c \sin \chi)\left(1+\frac{1}{4} \kappa-\frac{5}{8} \kappa \cos \gamma\right)+(b-c \sin \chi) \frac{5}{8} \kappa \sin \gamma,
\end{aligned}
$$

where

$$
\begin{aligned}
& a=-e_{J} \frac{A_{2}}{A_{0}}, \quad b=-e_{J} \frac{A_{1}}{A_{0}}, \quad c=\varepsilon \rho_{11}, \quad \kappa=\varepsilon \lambda_{0}^{2}, \\
& \chi=A_{0} \tau+\psi_{11}, \quad \gamma=A_{3} \tau+2 A_{4} w-2 \varpi_{J}-2 \nu_{02},
\end{aligned}
$$

and $\rho_{11}, \psi_{11}, \lambda_{0}, \nu_{02}$ are constants.The letters $A_{i}$ denote known expressions, given in (Érdi, 1981, 1984), of a parameter $l$ which characterizes the amplitude of libration around $L_{4}$. The parameter $\kappa$ is connected with the orbital inclination $i, i=\sqrt{\kappa}+O(\varepsilon)\left(i=0\right.$ for $\left.\lambda_{0}=0\right)$.

The long-term variations of $e_{J}$ and $\varpi_{J}$ can be described by the equations (Bretagnon, 1974)

$e_{J} \cos \varpi_{J}=\sum_{j=1}^{8} \lambda_{J j} M_{j} \cos \left(g_{j} t+\beta_{j}\right), \quad e_{J} \sin \varpi_{J}=\sum_{j=1}^{8} \lambda_{J j} M_{j} \sin \left(g_{j} t+\beta_{j}\right)$,

where $\lambda_{J j}, M_{j}, \beta_{j}$ are constants and $g_{j}$ are the secular fundamental frequencies. 

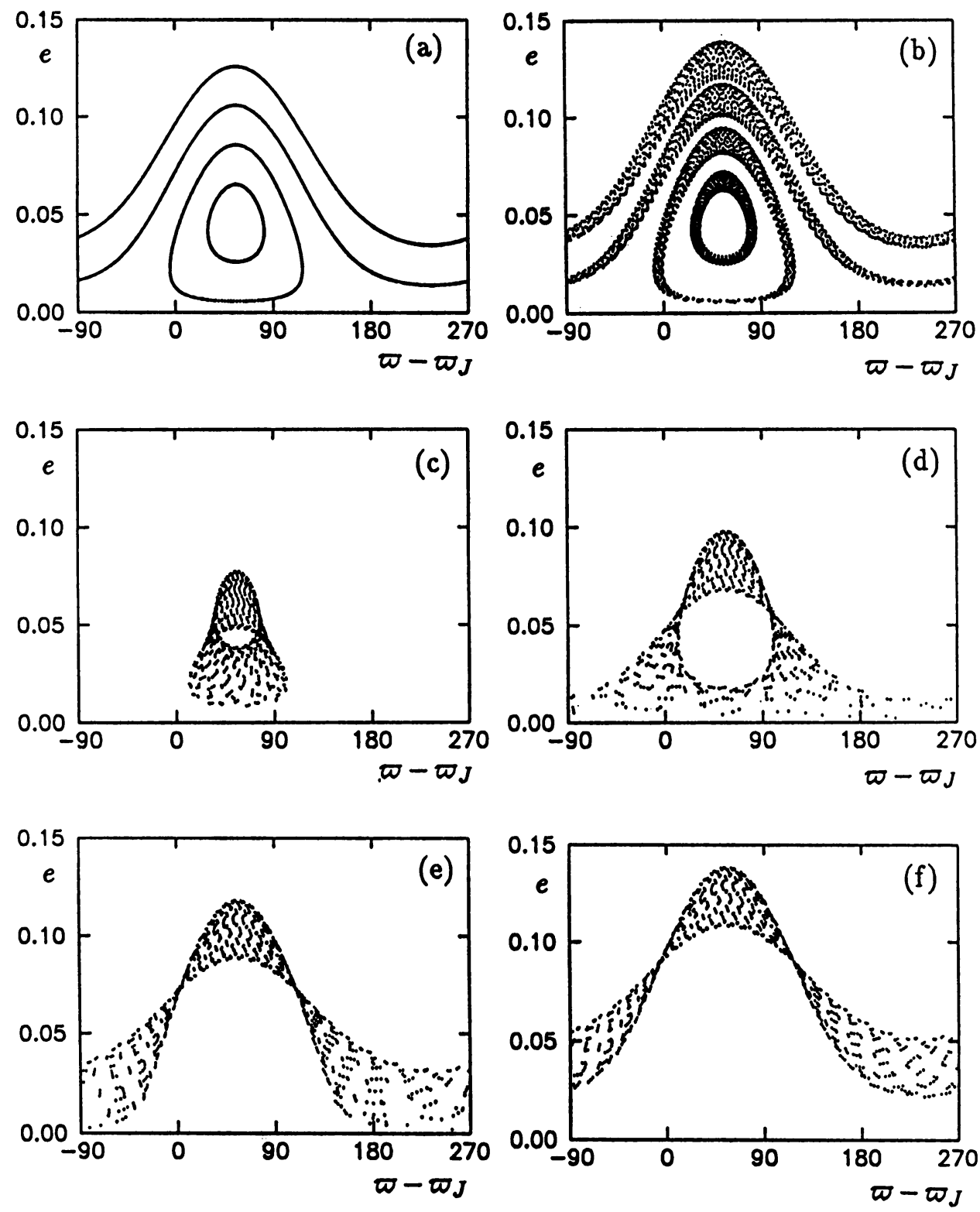

Figure 1. Variation of $e$ versus $\varpi-\varpi_{J}$. (a): zero inclination orbits, $e_{J}$ constant; (b): inclined orbits, $e_{J}$ constant; (c)-(f): zero inclination orbits, $e_{J}$ secularly changing. 
Fig. 1 shows $e$ versus $\varpi-\varpi_{J}$ for several values and cases of the parameters of Equations (4). In all examples $l=0.3$ (libration amplitude $17.2^{0}$ around $\left.L_{4}\right), \psi_{11}=0, \nu_{02}=0$, and the investigated time interval is 150000 years.

Fig. 1(a) is obtained for $\lambda_{0}=0(i=0), e_{J}=0.048$, and for several values of $c: c=0.02,0.04,0.06,0.08$. The inner closed curve corresponds to $c=0.02$, and $c$ is increasing from orbit to orbit outwards. In this case there is only a free oscillation of the perihelion with proper frequency $\varepsilon^{2} A_{0} n_{J}$, and $e$ and $\varpi-\varpi_{J}$ change with periods of the order of 3600 years. Note that the centre $e=e_{J}, \varpi-\varpi_{J}=60^{\circ}$ corresponds to Lagrange's equilibrium solution. Fig. 1(a) is in good agreement with the result of Yoshikawa (1989, Fig. 10).

Fig. 1(b) is obtained for $\lambda_{0}=2\left(i \approx 19^{0}\right), e_{J}=0.048$, and for the same values of $c$ as in Fig. 1(a). The inclusion of the inclination dependent terms in Equations (4) brings in several long periods and the result is the broadening of the former simple curves of Fig. 1(a).

Figs. 1(c)-1(f) are obtained for $\lambda_{0}=0(i=0)$ and $e_{J}$ changing: $c=0.02$ for Fig. 1(c), $c=0.04$ for Fig. 1(d), $c=0.06$ for Fig. 1(e), $c=0.08$ for Fig. 1(f). The secular variation of $e_{J}$ broadens the limits of the variation of the eccentricity and may cause the alternation of libration and circulation of the perihelion (Fig. 1(d)). When $\lambda_{0} \neq 0$, that is for inclined orbits, the regions of Figs. 1(c)-1(f) have further broadening (not shown here), however the dominant effects in the long-term variation of the eccentricity of the Trojans come from the secular change of the eccentricity of Jupiter.

\section{References}

Bien, R. and Schubart, J.: 1987, Astron. Astrophys. 175, 292.

Bretagnon, P.: 1974, Astron. Astrophys. 30, 141.

Érdi, B.: 1981, Celest. Mech. 24, 377.

Érdi, B.: 1984, Celest. Mech. 34, 435.

Érdi, B.: 1988, Celest. Mech. 43, 303.

Garfinkel, B.: 1985, Celest. Mech. 36, 19.

Kevorkian, J.: 1970, In Periodic Orbits, Stability and Resonances (G. E. O. Giacaglia ed.) D. Reidel Publ. Co. Dordrecht, Holland, 286.

Milani, A.: 1993, Celest. Mech. 57, 59.

Milani, A.: 1994, In Asteriods, Comets, Meteors 1993 (A. Milani et al. eds.), 1994 IAU Printed in the Netherlands, 159.

Zagretdinov, R. V.: 1986, Kinematika i Fizika Nebesnih Tel 2 N 3, 68.

Yoshikawa, M.: 1989, Astron. Astrophys. 213, 436. 